\title{
Adherence to oral oncolytics filled through an internal health-system specialty pharmacy compared with external specialty pharmacies
}

\author{
Emmeline C Academia, PharmD, BCOP; Caroline M Mejías-De Jesús, PharmD, BCOP; Julia S Stevens, PharmD, BCOP; \\ Lily Y Jia, PharmD, BCOP; Tuyen Yankama, MPH; Chirag Patel, MS; and Jinkyu Lee, PharmD, BCACP, CSP
}

\section{What is already known about this subject}

- Oral oncolytics are becoming increasingly common in the treatment of solid and hematological malignancies, requiring adherence at home to ensure effective treatment.

- Internal health-system specialty pharmacies (HSSPs) have been reported to improve medication adherence, but further data are needed to confirm this in a broader oncology population.

- Some studies have demonstrated shorter time to treatment with internal HSSPs.

\section{What this study adds}

- By confirming positive effects of internal HSSPs on oral oncolytic adherence, this study provides pharmacy and health-system leadership with additional proportion of days covered, medication possession ratio, and time-to-treat data supporting the benefits of internal HSSPS.

- Promotion of internal pharmacy prescription capture is a valid means to potentially optimize adherence outcomes.

\section{Author affiliations}

Emmeline C Academia, PharmD, BCOP; Caroline M Mejías-De Jesús, PharmD, BCOP; Julia S Stevens, PharmD, BCOP; Lily Y Jia, PharmD, BCOP; and Jinkyu Lee, PharmD, BCACP, CSP, Department of Pharmacy, Ambulatory Clinical Pharmacy, Beth Israel Deaconess Medical Center, Boston, MA. Tuyen Yankama, MPH, and Chirag Patel, MS, Department of Pharmacy, Pharmacy Data Analytics, Beth Israel Deaconess Medical Center, Boston, MA.

\section{AUTHOR CORRESPONDENCE:}

Emmeline C Academia, eacademi@bidmc.harvard.edu

J Manag Care Spec Pharm 2021;27(10):1438-46

Copyright $\odot 2021$, Academy of Managed Care Pharmacy. All rights reserved.

\section{ABSTRACT}

BACKGROUND: Oral oncolytics are becoming increasingly common in the treatment of solid and hematological malignancies. Medication adherence is especially important to ensure adequate drug levels to treat active malignancies, notably in curativeintent therapy. Further data are needed to quantify and confirm the effects of internal health-system specialty pharmacies (HSSPs) on medication adherence.

OBJECTIVE: To confirm the effect of an internal HSSP compared with external specialty pharmacies on oncolytic adherence as measured by proportion of days covered (PDC), medication possession ratio (MPR), and time to treatment (TTT).

METHODS: This single-center retrospective cohort study included patients receiving oral oncolytics through an internal HSSP or external specialty pharmacies between January 2019 and June 2020. Fill data were extracted from pharmacy claims databases and electronic medical records. The primary adherence outcome was patient-level PDC. Secondary adherence outcomes included patient-level MPR and TTT. For PDC and MPR analyses, patients with at least 3 fills per oncolytic were included. All patients were included for the TTT analysis. Chi-square or Fisher's exact tests were used to analyze categorical differences between pharmacy groups. Differences in continuous variables across pharmacy groups were evaluated using Wilcoxon rank-sum tests.

RESULTS: 871 prescriptions met inclusion criteria: 549 patients were included in the PDC/ MPR analysis, and 758 patients were included in the TTT analysis (patients might have multiple prescriptions). Patients who filled at an internal HSSP had a higher median PDC compared with those who filled at external specialty pharmacies $(0.99[\mathrm{IQR}=0.89$ 1.00 ] vs 0.91 [IQR=0.76-0.98]; $P<0.01$ ). The 
adherence rate as measured by MPR was higher for patients who used an internal HSSP compared with those who used external specialty pharmacies (MPR $=1.00$ [IQR $=0.90-1.00]$ vs 0.93 [IQR $=0.76-1.00]$; $P<0.01$ ). Median TTT was lower for patients using the internal HSSP vs an external specialty pharmacy (5 days [IQR $=2-13]$ vs 27 days [IQR=2-82], respectively; $P<0.01$ ).

CONCLUSIONS: Internal HSSP services improved adherence as measured by PDC and MPR. Significantly lower TTT was seen with the internal HSSP compared with external pharmacies. These data confirm and support use of internal HSSPs to dispense oral oncolytics for treatment of solid and hematological malignancies.

Oral oncolytics are becoming increasingly more common in the treatment of various malignancies. Of over 800 new oncology therapies in the drug development pipeline, $25 \%$ $35 \%$ are oral agents. ${ }^{1}$ With more oral therapies in oncology, medication adherence is particularly important to ensure adequate drug levels to treat active malignancies, especially in curative-intent therapy. Nonadherence may reduce time to progression and decrease overall survival. In chronic myeloid leukemia (CML), poor adherence to oral tyrosine kinase inhibitors (TKIs) has been associated with failure to obtain molecular responses and maintain event-free survival. ${ }^{2}$ In outpatient and inpatient/emergency settings, adherence to TKI therapy in CML patients has also been shown to reduce risk of all-cause health care utilization. ${ }^{3}$ In non-small cell lung cancer patients starting erlotinib TKI therapy, patients with low-income subsidy status, previous use of intravenous chemotherapy, and increased patient out-of-pocket costs were associated with decreased adherence to erlotinib. ${ }^{4}$ However, rates of adherence to oral oncolytics have varied greatly in the literature, ranging from $46 \%$ to $100 \%{ }^{5}$

Adherence is commonly measured by medication possession ratio (MPR) and proportion of days covered (PDC), ${ }^{6}$ definitions of which are discussed further in the Methods section. PDC is the adherence measure used by the Centers for Medicare \& Medicaid Services star ratings that measure Part C and Part D performance data. ${ }^{7}$ The Utilization Review Accreditation Commission (URAC) also selected PDC as a mandatory measure for accreditation, defining adherence with a PDC goal of $80 \%$ or more, with the exception of a goal of $90 \%$ for antiretrovirals. ${ }^{8}$ Another metric used by specialty pharmacies is time to treatment (TTT), defined as the number of days between the prescribing date and the first fill date, which determines initiation of treatment and can be clinically relevant in patients who need rapid disease control. $^{9}$
The benefits of using internal health-system specialty pharmacies (HSSPs) continue to be elucidated using measures of PDC, MPR, and TTT. Potential advantages of internal specialty pharmacy services include faster receipt of medication, prevention of medication errors, increased patient and provider satisfaction, and better management of adherence and side effects, promoting high quality care of cancer patients. ${ }^{10}$ Farano et al demonstrated the effectiveness of an oncology specialty pharmacy practice in an internal HSSP, reporting cost savings of over $\$ 300,000$ to patients and an average time to insurance approval of 1.2 days for a cohort of 75 patients. ${ }^{11}$ In a study by McCabe et al, patients who filled their oral oncolytic prescriptions at an internal HSSP had higher adherence as measured by MPR and PDC, compared with external specialty pharmacies $(1.00$ vs $0.75 ; \mathrm{P}<0.001$ and 0.95 vs $0.70 ; \mathrm{P}<0.001$, respectively). ${ }^{9}$ Shorter TTT was also seen with internal HSSP services ( 6.85 vs 10.91 days; $P=0.102){ }^{9}$

The objective of this study was to confirm the effect of an internal HSSP compared with external specialty pharmacies in a larger solid and hematologic oncology population than previously reported, while also validating previous findings. Adherence was measured primarily by PDC, with secondary outcomes measuring MPR and TTT, using multiple methods to compute PDC and MPR to confirm findings.

\section{Methods}

\section{STUDY DESIGN}

This single-center retrospective cohort study included patients receiving eligible oral oncolytics prescribed by solid and hematologic oncology providers at Beth Israel Deaconess Medical Center (BIDMC) in Boston, Massachusetts, between January 2019 and June 2020 (Supplementary Table 1, available in online article). MPR is calculated as the number of days supply dispensed divided by the number of days in the time period of interest. A value of 1 corresponds to $100 \%$ adherence, while a value less than 1 indicates underadherence. Overestimation of adherence (MPR $>1.00)$ can occur because of early refills. ${ }^{6}$ PDC is calculated as the number of days covered divided by the number of days in the time period of interest, with a value of 1 corresponding to $100 \%$ adherence. The PDC calculation adjusts for overlapping supply and cannot exceed $100 \%{ }^{6}$ Given the URAC selection of PDC as a mandatory measure for accreditation, and PDC goal of $80 \%$ or more for adherence, a definition of PDC greater than $80 \%$ was used to classify patients as high adherence. TTT is defined as the number of days between the prescribing date and the first fill date, which determines initiation of treatment. ${ }^{9}$ 


\section{FIGURE 1 Flowchart of Patient Enrollment}

Between January 1, 2019, and June 30, 2020:

883 electronic prescriptions assessed

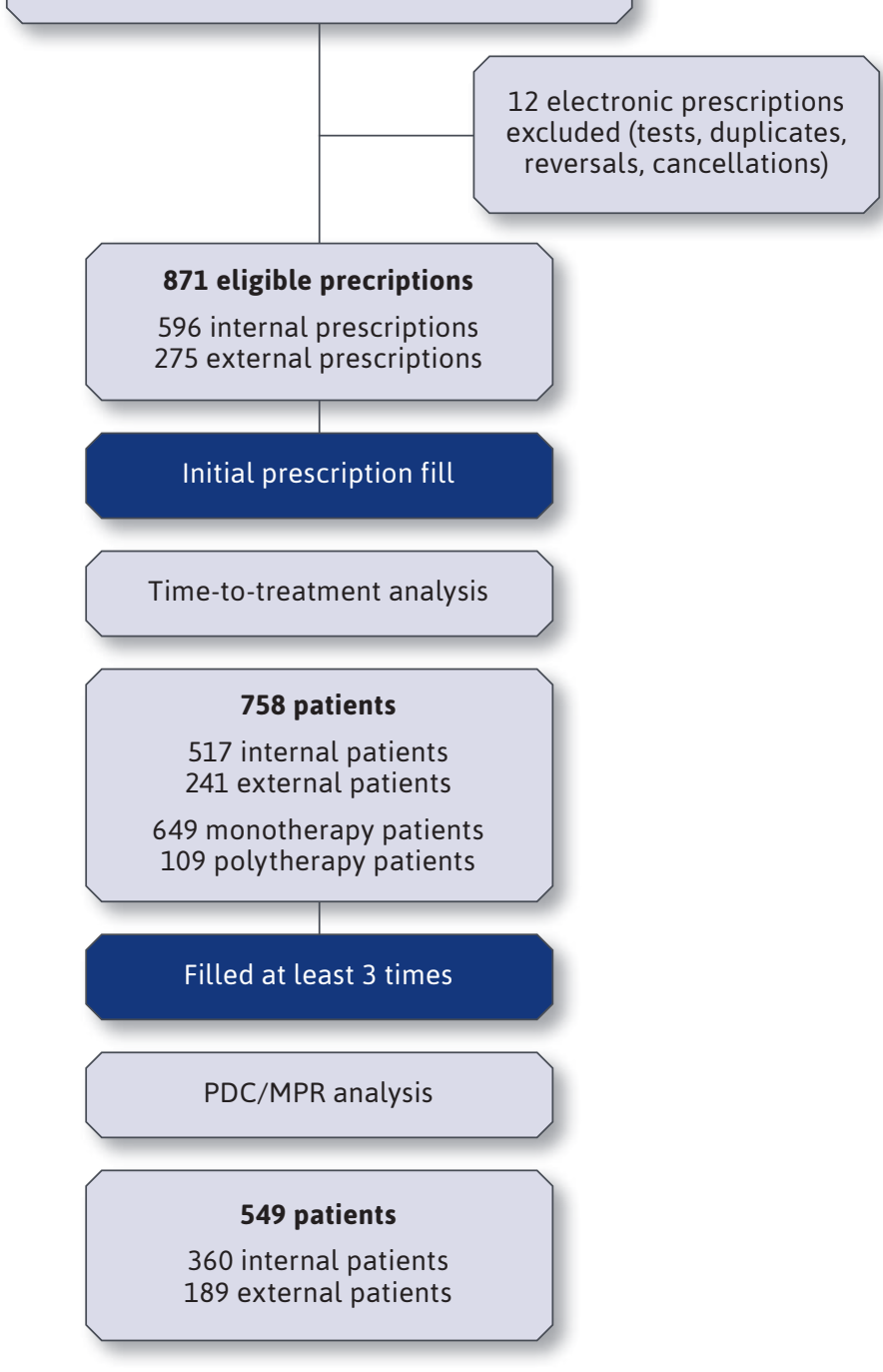

$M P R=$ medication possession ratio; $P D C=$ proportion of days covered .

For the PDC and MPR calculations, only patients with 3 or more claims per oncolytic were included (Figure 1 ). The requirement of 3 claims provided at least 2 time periods for adherence assessments (between initial and first claim and again between first and second claim), whereas the requirement of 2 claims only provided 1 time period. For patients with only 2 claims, their overall adherence as assessed via PDC/MPR would not be as representative as those with 3 or more claims. All patients were included for the
TTT analysis. Fill data were extracted from the pharmacy claims database and external databases from participating external specialty pharmacies with which the internal HSSP was contracted. Patient demographics, insurance, and diagnosis information were extracted from electronic medical records.

Prescriptions provided via fax, telephone, and paper were excluded. Test claims, duplicate scripts, and prescriptions returned to the pharmacy (reversed or cancelled) were removed. Patient demographic information was also collected, including age, race, sex, insurance type, and primary diagnosis from the most recent clinic visit. Data were collected approximately 6 months after the latest patient-level first fill date. Since the study was initiated for quality improvement purposes, and due to its retrospective nature and minimal risk, the BIDMC Institutional Review Board approved this study with an informed consent waiver.

\section{OUTCOMES}

The primary adherence outcome of patient-level PDC was calculated based on whether patients filled 1 medication (oral oncolytic monotherapy) or multiple concurrent medications (oral oncolytic combination therapy, polytherapy) throughout the study period. For patients receiving monotherapy, early refills were accounted for to avoid overlap medication coverage, prorating to start on the day after exhaustion of the previous supply (Supplementary Figure 1A, available in online article). For patients on polytherapy, PDC was calculated per medication, and average PDC was computed per patient. The secondary adherence outcome was patientlevel MPR. For patients on polytherapy, MPR was calculated for each medication, and an average MPR was computed for each patient. MPR was capped at 1 or $100 \%$.

For PDC and MPR calculations, the patient-level index date was defined as the first fill date, and the time period of interest was defined as the duration between the first and the last fill dates (prescription-based measure). Medication supply that fell outside of the study period was truncated. The secondary outcome of TTT was calculated based on the initial fill date and date prescribed, which was used as the surrogate marker for patient receipt of the medication. Differences in the proportion of patients classified as high adherence, defined as $\mathrm{PDC}>80 \%$ or $\mathrm{MPR}>80 \%$, were also determined.

\section{STATISTICAL ANALYSIS}

Descriptive statistics were used to characterize the study population. Continuous variables, which were skewed by extreme values, were reported as medians and interquartile ranges (IQRs), with differences between internal and external specialty pharmacies tested using the Wilcoxon 
rank-sum test. Categorical variables were reported as counts with frequencies, with differences between groups tested using chi-square or Fisher's exact tests, as appropriate. PDC was reported as a continuous and a dichotomized measure. Differences in median PDC across pharmacy groups were evaluated using the Wilcoxon rank-sum test. Differences in the proportion of patients with high adherence between internal HSSPs and external specialty pharmacies were evaluated using chi-square tests. Similar analyses were performed to compare MPR and TTT across groups, with TTT being reported only as a continuous measure.

There was no standardized end date definition for PDC/ MPR calculation and no standardized method to calculate PDC/MPR for patients with 2 or more oncolytics, which can lead to wide variability in adherence estimates. ${ }^{12} \mathrm{We}$ performed preplanned sensitivity analyses on PDC and MPR measures to assess the robustness of our results. We tested a different definition of end date, calculating PDC and MPR with an end date defined as June 2020 (interval-based measure).

In addition, we tested 2 additional approaches in calculating PDC for patients on polytherapy. In the first PDC sensitivity analysis, all patients who filled at least 2 oral oncolytics were assumed to be on polytherapy. PDC was defined as days on which all medications were available (Supplementary Figure 1B, available in online article). In the second PDC sensitivity analysis, patients who filled at least 2 oral oncolytics were confirmed to be on combination therapy by ambulatory hematology/oncology pharmacists; PDC was then defined as days on which all oral oncolytics were available.

For patients who filled at least 2 oral oncolytics and were not confirmed to be on combination therapy (eg, deemed to be separate treatment lines), PDC was calculated per medication, and average PDC was computed per patient. The study sample size was expected to vary, since different methods of handling patients on polytherapy were evaluated because of exclusion of medications with less than 3 fills. For example, a patient had 2 claims of oncolytic A and 1 claim of oncolytic B. This patient would be excluded from the primary analysis, which required patients with at least 3 fills for each oncolytic. In our sensitivity analyses of PDC calculation, this patient would be included, since PDC can be computed from all 3 claims.

Additional sensitivity analyses were performed on patients with PDC less than 50\%. These patients were reviewed by hematology/oncology clinical pharmacists to identify whether low PDC could be attributed to nonadherence or whether there were other reasons for extended gaps in therapy. Reasons for extended gaps included intentional holds for adverse drug reactions, prolonged hospitalizations, drug holidays, and fill history discrepancies. The first analysis was performed excluding patients with low PDC due to circumstances other than nonadherence. The second analysis focused on patients who filled at the internal HSSP and at an external specialty pharmacy and had at least 2 fills (1 initial and 1 refill).

Assuming all baseline characteristics remained constant, focusing on this cohort might offer a less confounded insight on the difference in outcomes between the 2 specialty pharmacy groups. The cohort of patients filling at the HSSP and external pharmacies was very small. By relaxing the requirement for number of fills from 3 to 2, we were able to perform this sensitivity analysis. Differences in median PDC were evaluated using the Wilcoxon signed-rank test. Differences in the proportion of patients classified as high adherence, which was defined as PDC greater than $80 \%$, between internal HSSP and external specialty pharmacies were evaluated using McNemar's test. Methods to handle PDC calculation for polytherapy patients were previously defined in the Outcomes section.

All tests were 2-sided, and the nominal level of statistical significance ( $\alpha$ ) was $5 \%$. Analyses were performed using R version 3.6.3 (R Foundation for Statistical Computing).

\section{Results}

\section{PATIENT CHARACTERISTICS}

Of the 871 prescriptions that met the inclusion criteria, 549 patients were included in the PDC/MPR analysis, since they met the inclusion criteria of having at least 3 fills (Figure 1). For the TTT analysis focusing on the initial fill, 758 patients were included (each patient might have more than 1 prescription). Among 758 patients, 649 were on monotherapy and 109 were on polytherapy. Patients with less than 3 fills were excluded from the PDC/MPR analysis but were included in the TTT analysis. The baseline characteristics of our study sample are summarized in Table 1. In both PDC/ MPR and TTT analyses, patients who filled at the internal HSSP were older (median age 72 years) than those who filled at external specialty pharmacies (median age 63-64 years; $P<0.01)$. The majority of patients were White and had Medicare insurance. The primary diagnoses varied significantly between the internal HSSP and external specialty pharmacies, with differences largely in gastrointestinal (11\% vs $3 \%$ ), gynecologic ( $7 \%$ vs $1 \%)$, other ( $1 \%$ vs $6 \%$ ), and unknown (1\% vs $13 \% ; \mathrm{P}<0.01)$.

\section{ADHERENCE BY PDC}

Patients who filled at an internal HSSP had significantly higher median PDC compared with those who filled at 


\section{TABLE 1 Baseline Characteristics Stratified by Internal and External Specialty Pharmacy Use}

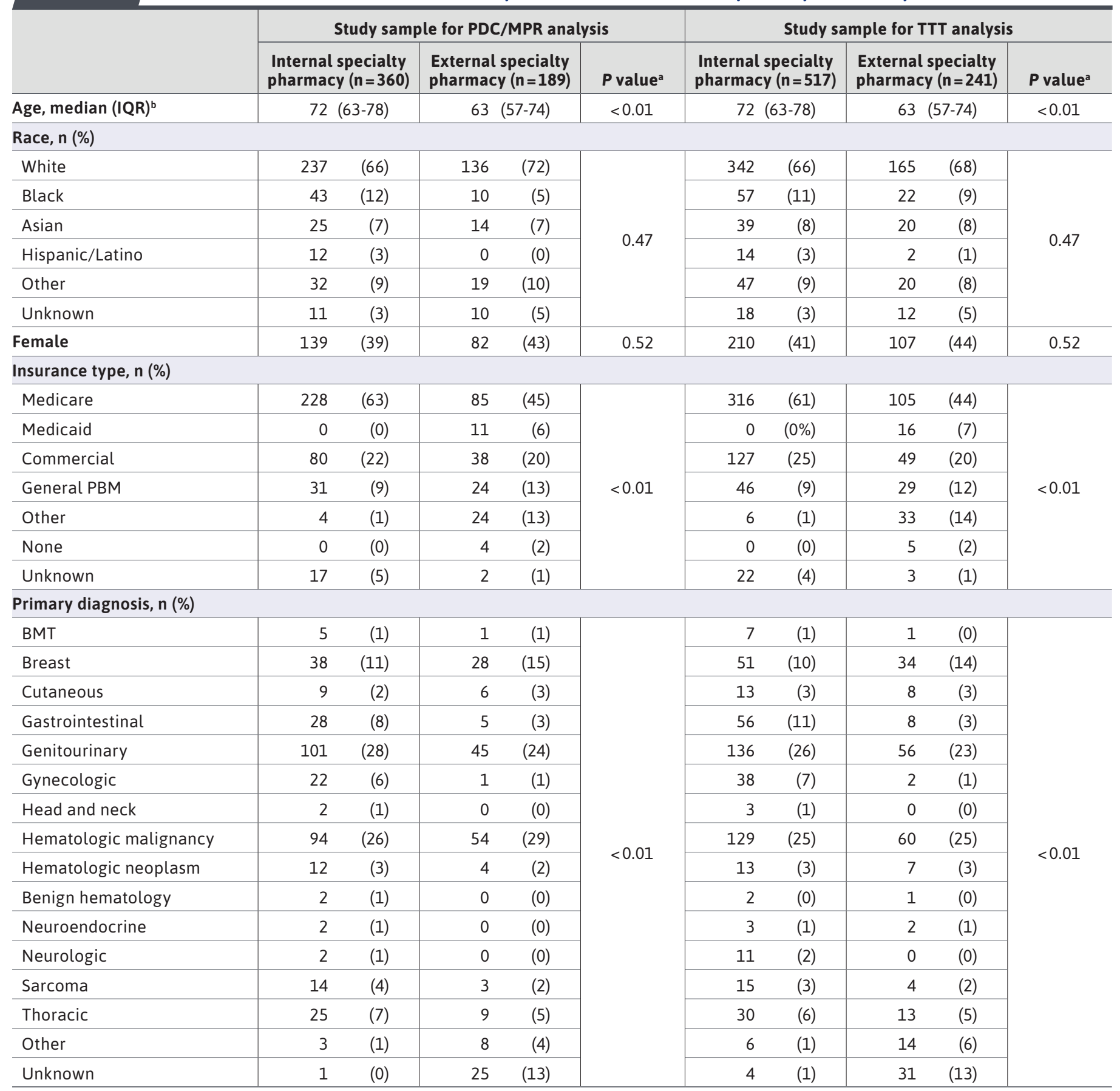

ap values were calculated using either chi-square or Fisher's exact tests, unless otherwise specified.

${ }^{b}$ Medians and IQRs were tested using the Wilcoxon rank-sum test.

$B M T=$ bone marrow transplant; $I Q R=$ interquartile range; $M P R=$ medication possession ratio; $P B M=$ pharmacy benefit manager; $P D C=$ proportion of days covered; TTT = time to treatment. 


\section{FIGURE 2 PDC Stratified by Internal and External Specialty Pharmacy Use}

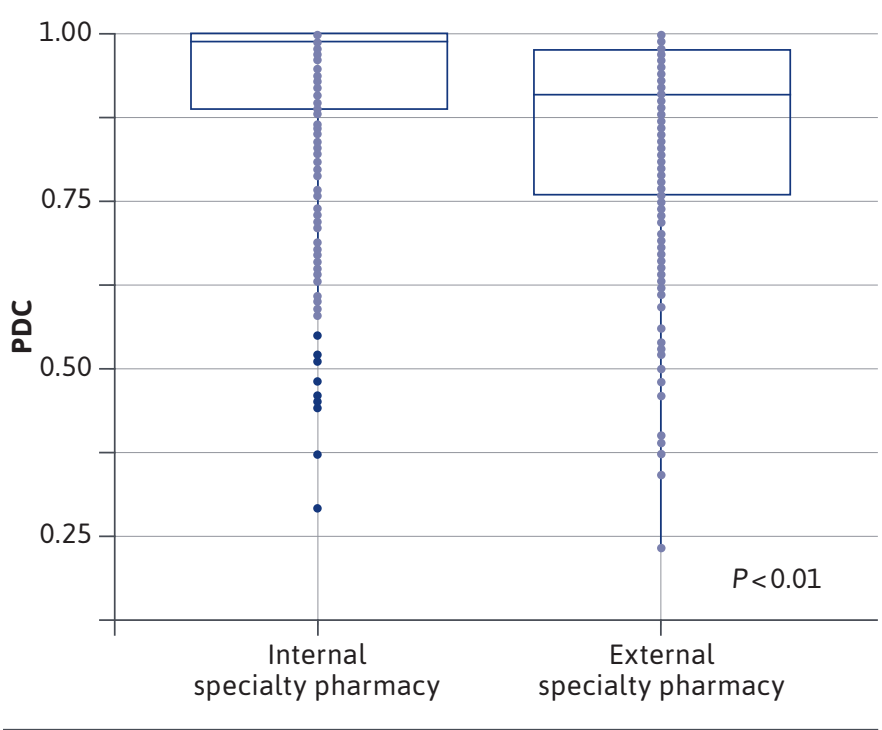

Light blue circles: Data points that are not outliers

Dark blue circles: Data points that are potential outliers or those outside the range of 25th percentile

$P D C=$ proportion of days covered.

external specialty pharmacies $(0.99$ [IQR $=0.89-1.00]$ vs 0.91 [IQR=0.76-0.98], respectively; $\mathrm{P}<0.01$ ). Figure 2 displays the difference in PDC across the 2 groups. There was a higher proportion of patients who were classified as high adherence, with PDC $>80 \%$, among those who used the internal HSSP compared with those who used external specialty pharmacies ( $83 \%$ vs $69 \%$, respectively; $\mathrm{P}<0.01$; Figure 2 and Table 2).

Differences in adherence by PDC were still observed despite the varying estimates when analyzed with a different end date definition and methods of handling polytherapy in the PDC calculation (Table 3). The median PDC of patients filling with the internal HSSP ranged between 0.97 and 0.99 , with PDC greater than $80 \%$ between $70 \%$ and $82 \%$. External specialty pharmacy median PDC ranged between 0.89 and 0.91 , with PDC greater than $80 \%$ between $64 \%$ and $69 \%$. When calculated via interval-based measure, PDC estimates were lower. For patients using the internal HSSP, median PDC with this method was between 0.73 and 0.84; PDC greater than $80 \%$ was between $46 \%$ and $52 \%$. With external specialty pharmacies, the median PDC was between 0.58 and 0.61 , with PDC greater than $80 \%$ ranging from $29 \%$ to $31 \%$. The difference in PDC between internal vs external specialty pharmacies remained significant across all sensitivity analyses $(\mathrm{P}<0.01$; Table 3$)$.

\section{ADHERENCE BY MPR}

The adherence rate as measured by median MPR was significantly higher for patients who used the internal HSSP compared with those who used external specialty pharmacies $(\mathrm{MPR}=1.00 \quad[\mathrm{IQR}=0.90-1.00]$ vs 0.93 [ $\mathrm{IQR}=0.76-1.00]$, respectively; $\mathrm{P}<0.01)$. More patients also met criteria for high adherence per MPR when filling with the internal HSSP compared with external pharmacy fills ( $85 \%$ vs $71 \%$, respectively; $\mathrm{P}<0.01)$. While, as expected, changing the end date definition for MPR calculation substantially reduced estimates, the difference in adherence to oral oncolytics filled with an internal pharmacy remained when analyzing for median MPR (0.85 [IQR=0.49-1.00] vs 0.61 [IQR=0.40-0.86], respectively; $\mathrm{P}<0.01)$ and MPR greater than $80 \%(53 \%$ vs $31 \%$, respectively; $\mathrm{P}<0.01$; Table 3 ).

Of 549 patients, 20 (4\%) had PDC less than 50\% and were reviewed for extended gaps in therapy. These patients are represented by dark blue dots in Figure 2. Of the 20 cases, 7 (35\%) filled internally, and 13 (65\%) filled externally. Reasons for extended gaps included adverse drug reaction (40\%), data inaccuracy (20\%), suspected nonadherence $(10 \%)$, drug holiday (10\%), documented nonadherence (10\%), discrepancy between fill date and start date (5\%), and no documentation (5\%). After excluding patients with low PDC unrelated to nonadherence, adherence rate per PDC was still higher among those who filled internally compared with those who filled externally $(0.99[\mathrm{IQR}=0.90-1.00]$ vs $0.92[\mathrm{IQR}=0.79-0.99]$, respectively; $\mathrm{P}<0.01$ ). The proportion of patients with high adherence per PDC remained significantly different when comparing patients who filled internally and externally ( $85 \%$ vs $72 \%$, respectively; $P<0.01)$.

There were 11 patients who filled both at the internal HSSP and an external specialty pharmacy. For these patients, the difference in PDC was not statistically significant (median PDC: 0.94 [IQR=0.74-1.00] vs 0.90 [IQR=0.65-0.98], respectively, $\mathrm{P}=0.61$; $\mathrm{PDC}>80 \%$ : $55 \%$ vs $55 \%$, respectively, $\mathrm{P}=1.00$ ). There was also no statistically significant difference in MPR (median MPR: 0.96 [IQR=0.74-1.00] vs 0.98 [IQR=0.65-1.00], respectively, $\mathrm{P}=0.69$; $\mathrm{MPR}>80 \%$ : $64 \%$ vs $55 \%$, respectively, $\mathrm{P}=0.77)$. The TTT was shorter for patients who filled internally (1.5 days $[\mathrm{IQR}=1-6]$ vs 30 days [IQR=4-82]; $\mathrm{P}=0.08$ ).

\section{TIME TO TREATMENT}

Median TTT was significantly lower for patients who used an internal HSSP compared with an external specialty pharmacy ( 5 days [ $\mathrm{IQR}=2-13]$ vs 27 days [ $\mathrm{IQR}=2-82]$, respectively; $\mathrm{P}<0.01$; Table 2). 


\begin{tabular}{|c|c|c|c|c|c|c|}
\hline Outcome & Time frame & Method for polytherapy & Measurements & $\begin{array}{c}\text { Internal specialty } \\
\text { pharmacy }\end{array}$ & $\begin{array}{c}\text { External specialty } \\
\text { pharmacy }\end{array}$ & $P$ value \\
\hline \multirow[t]{3}{*}{ PDC } & \multirow{3}{*}{$\begin{array}{l}\text { Prescription-based } \\
\text { measure }\end{array}$} & \multirow{3}{*}{$\begin{array}{l}\text { Calculate PDC per } \\
\text { medication and average } \\
\text { PDC per patient }\end{array}$} & Sample size & 360 & 189 & - \\
\hline & & & Median (IQR) & $0.99(0.89-1.00)$ & $0.91(0.76-0.98)$ & $<0.01$ \\
\hline & & & $\mathrm{PDC}>80 \%^{\mathrm{a}}$ & $299(83 \%)$ & $130(69 \%)$ & $<0.01$ \\
\hline \multirow[t]{3}{*}{ MPR } & \multirow{3}{*}{$\begin{array}{l}\text { Prescription-based } \\
\text { measure }\end{array}$} & \multirow{3}{*}{$\begin{array}{l}\text { Calculate MPR per } \\
\text { medication and average } \\
\text { MPR per patient }\end{array}$} & Sample size & 360 & 189 & - \\
\hline & & & Median (IQR) & $1.00(0.90-1.00)$ & $0.93(0.76-1.00)$ & $<0.01$ \\
\hline & & & $\mathrm{MPR}>80 \%^{\mathrm{a}}$ & 305 (85\%) & $134(71 \%)$ & $<0.01$ \\
\hline \multirow[t]{2}{*}{ TTT, days } & \multirow{2}{*}{$\begin{array}{l}\text { Prescribing date to } \\
\text { first fill date }\end{array}$} & \multirow{2}{*}{$\begin{array}{l}\text { Calculation only for } \\
\text { initial fill }\end{array}$} & Sample size & 517 & 241 & - \\
\hline & & & Median (IQR) & $5(2-3)$ & $27(2-82)$ & $<0.01$ \\
\hline
\end{tabular}

${ }^{a}$ Definition of high adherence.

$I Q R=$ interquartile range; $M P R=$ medication possession ratio; $P B M=$ pharmacy benefit manager; $P D C=$ proportion of days covered; $T T T=$ time to treatment.

\section{Discussion}

Results of this large dataset further confirm improvements in adherence with the use of an internal HSSP in an oncology population. As oral oncolytics become increasingly central to cancer treatment, efforts promoting patient adherence to therapy are paramount to optimizing clinical outcomes. We confirmed improved adherence with an internal HSSP using PDC and MPR, while also demonstrating significantly lower TTT.

Specialty pharmacy services, especially those provided internally within a health system, have been frequently associated with better outcomes. In 2010, a National Comprehensive Cancer Network task force report listed potential advantages of specialty pharmacy services, which included appropriate medication selection, increased adherence, avoidance of unnecessary drug costs, and increased patient and provider satisfaction. ${ }^{10}$ Additionally, disease state complexities and treatment toxicities highlight the need for integrated clinical and pharmacy services to ensure safe and efficient treatment, while fostering better interdisciplinary collaboration..$^{13,14}$

Internal HSSPs have also been found to prevent prescription errors and improve toxicity monitoring. $1{ }^{15}$ Furthermore, fully integrated specialty pharmacies in health systems have reported higher prior authorization approvals, higher medication adherence, and overall greater patient satisfaction. ${ }^{13}$ Consistent with these findings, the current study confirms reduced TTT, which highlights the inherent benefits of an integrated, internal HSSP system that is able to address prior authorization requirements and collaborate directly with the treating team. Reduced access to the medical record and treatment teams inherently increase
TTT when prescriptions are filled at external specialty pharmacies. Taken together, improvements in the fulfillment of oral oncolytics in an internal HSSP may improve patient outcomes, especially in a solid and hematological oncology population.

Poor adherence has been associated with poorer health outcomes and increased health care utilization costs. ${ }^{2,3}$ It is thus important to promote high adherence to medications, as defined by either PDC or MPR greater than $80 \%$. A threshold of PDC greater than $80 \%$ is the goal for adherence set forth by URAC, the accrediting body. Consistent with previously published reports, our large dataset of 549 patients in the PDC/MPR analysis and 758 patients in the TTT analysis demonstrate improvements in adherence, with significantly higher PDC compared with those filled at external specialty pharmacies. These results are confirmed with improvements in multiple adherence measures and additional sensitivity analyses.

Some differences in adherence to oral oncolytics filled at internal HSSPs and external specialty pharmacies were seen, for example, in gastrointestinal (11\% vs 3\%, respectively) and gynecologic (7\% vs 1\%, respectively) malignancies. A paucity of data exists to explain some of the differences, however, which could be attributed to age and toxicity of the oncolytics used in these disease states. Gastrointestinal malignancies tend to affect an older population, with the median age of diagnosis for colorectal cancer of 67 years and the median age of diagnosis for ovarian cancer of 63 years, for example. ${ }^{16,17}$ Older people have been found to have more risk factors for nonadherence, and include factors such as cognitive deficits, visual/hearing/functional impairment, and polypharmacy. ${ }^{18}$ 


\section{TABLE 3 Sensitivity Analyses Comparing Internal and External Specialty Pharmacy Use}

\begin{tabular}{|c|c|c|c|c|c|c|}
\hline Outcome & Time frame & Method for polytherapy & Measurements & $\begin{array}{c}\text { Internal specialty } \\
\text { pharmacy }\end{array}$ & $\begin{array}{c}\text { External specialty } \\
\text { pharmacy }\end{array}$ & $P$ value \\
\hline \multirow{15}{*}{ PDC } & \multirow{6}{*}{$\begin{array}{l}\text { Prescription- } \\
\text { based } \\
\text { measure }\end{array}$} & \multirow{3}{*}{ Treated as combination therapy } & Sample size & 367 & 196 & - \\
\hline & & & Median (IQR) & $0.97(0.72-1.00)$ & $0.89(0.69-0.97)$ & $<0.01$ \\
\hline & & & $\mathrm{PDC}>80 \%^{\mathrm{a}}$ & $257(70 \%)$ & $126(64 \%)$ & $<0.01$ \\
\hline & & \multirow{3}{*}{$\begin{array}{l}\text { Calculate PDC per medication and } \\
\text { average PDC per patient, unless } \\
\text { marked as combination therapy by } \\
\text { pharmacist }\end{array}$} & Sample size & 362 & 192 & - \\
\hline & & & Median (IQR) & $0.99(0.88-1.00)$ & $0.91(0.77-0.98)$ & $<0.01$ \\
\hline & & & $P D C>80 \%{ }^{a}$ & $296(82 \%)$ & $132(69 \%)$ & $<0.01$ \\
\hline & \multirow{9}{*}{$\begin{array}{l}\text { Interval- } \\
\text { based } \\
\text { measure }\end{array}$} & \multirow{3}{*}{$\begin{array}{l}\text { Calculate PDC per medication and } \\
\text { average PDC per patient }\end{array}$} & Sample size & 360 & 189 & - \\
\hline & & & Median (IQR) & $0.84(0.49-1.00)$ & $0.61(0.40-0.85)$ & $<0.01$ \\
\hline & & & PDC $>80 \%{ }^{a}$ & $188(52 \%)$ & $58(31 \%)$ & $<0.01$ \\
\hline & & \multirow{3}{*}{ Treated as combination therapy } & Sample size & 367 & 196 & - \\
\hline & & & Median (IQR) & $0.73(0.33-1.00)$ & $0.58(0.35-0.85)$ & $<0.01$ \\
\hline & & & $\mathrm{PDC}>80 \%^{\mathrm{a}}$ & $170(46 \%)$ & $57(29 \%)$ & $<0.01$ \\
\hline & & \multirow{3}{*}{$\begin{array}{l}\text { Calculate PDC per medication and } \\
\text { average PDC per patient, unless } \\
\text { marked as combination therapy by } \\
\text { pharmacist }\end{array}$} & Sample size & 362 & 192 & - \\
\hline & & & Median (IQR) & $0.82(0.47-1.00)$ & $0.61(0.40-0.85)$ & $<0.01$ \\
\hline & & & $\mathrm{PDC}>80 \%^{a}$ & $183(51 \%)$ & $59(31 \%)$ & $<0.01$ \\
\hline \multirow{3}{*}{ MPR } & \multirow{3}{*}{$\begin{array}{l}\text { Interval- } \\
\text { based } \\
\text { measure }\end{array}$} & \multirow{3}{*}{$\begin{array}{l}\text { Calculate MPR per medication and } \\
\text { average MPR per patient }\end{array}$} & Sample size & 360 & 189 & - \\
\hline & & & Median (IQR) & $0.85(0.49-1.00)$ & $0.61(0.40-0.86)$ & $<0.01$ \\
\hline & & & MPR $>80 \%{ }^{a}$ & $191(53 \%)$ & $59(31 \%)$ & $<0.01$ \\
\hline
\end{tabular}

aDefinition of high adherence.

$I Q R=$ interquartile range; $M P R=$ medication possession ratio; $P D C=$ proportion of days covered

Given these risks, closer follow-up with internal HSSPs may contribute to higher adherence rates. Gandhi et al reported results from a prospective, multicenter, surveybased study and found that patients who filled prescriptions at the pharmacy associated with the cancer center seemed to report toxicities more often than those not affiliated with a cancer center. ${ }^{19}$ They did, however, note that those filling prescriptions at a pharmacy outside of the cancer center tended to report toxicities to their family physicians or community pharmacists, rather than to a provider at the cancer center, suggesting that a lack of integration may contribute to poorer adherence. ${ }^{19}$

\section{LIMITATIONS}

Inherent limitations of this study include the retrospective nature of the study, which may affect the ability to limit confounding and to access complete datasets. As a single-center study, applicability to other institutions may be limited. Using time between first and last fill date as a retrospective surrogate to measure time on treatment (via PDC) may not accurately reflect a patient's treatment interval and may vary among institutions. This was mitigated through preplanned sensitivity analyses, including interval-based measures, alternate PDC analysis for patients on polytherapy, and review of patients with low PDC. Potential inaccuracies with this methodology may diminish the accuracy of any analysis attempting to identify other factors associated with adherence.

External pharmacies were reviewed in aggregate relative to the internal pharmacy, as such, it is difficult to know if some external pharmacies were performing as well as the internal HSSP. Other pharmacies may have contributed more to lower adherence numbers. Additionally, fill data used had the potential to be incomplete, since only data from our institution and contracted pharmacies were accessible and included. Other external data services were not used. Prospective studies in specific disease states, with live tracking of prescription fills and treatment holds, are needed to best capture nuances in fill dates and reasons for low PDC. 


\section{Conclusions}

Internal HSSP services were associated with significantly higher adherence compared with external specialty pharmacies, as measured by PDC and MPR. The internal HSSP pharmacy also achieved shorter TTT compared with prescriptions filled at an external pharmacy. These results were consistent across multiple sensitivity analyses and calculation methods. These data support the use of internal HSSPs for the fulfillment of oral oncolytics.

\section{DISCLOSURES}

This study received no financial support. The authors declare no potential conflicts of interest with respect to the research, authorship, and/or publication of this article.

\section{REFERENCES}

1. McBride A. The emerging role of oral oncolytics. Oncol Issues. 2020;35(1):3-3. doi:10.1080/10463356.2020.1706404.

2. Marin D, Bazeos A, Mahon F-X, et al. Adherence is the critical factor for achieving molecular responses in patients with chronic myeloid leukemia who achieve complete cytogenetic responses on imatinib. J Clin Oncol. 2010;28(14):2381-88. doi:10.1200/jco.2009.26.3087

3. Dashputre AA, Gatwood KS, Gatwood J. Medication adherence, health care utilization, and costs among patients initiating oral oncolytics for multiple myeloma or chronic lymphocytic leukemia/small lymphocytic lymphoma. J Manag Care Spec Pharm. 2020;26(2):186-96. doi:10.18553/ jmcp.2020.26.2.186

4. Hess LM, Louder, A, Winfree K, et al. Factors associated with adherence to and treatment duration of erlotinib among patients with non-small cell lung cancer. J Manag Care Spec Pharm. 2017;23(6):64352. doi: 10.18553/jmcp.2017.16389
5. Greer JA, Amoyal N, Nisotel L, et al. A systematic review of adherence to oral antineoplastic therapies. Oncologist. 2016;21(3):354-76.

6. Canfield SL, Zuckerman A, Anguiano RH, et al. Navigating the wild west of medication adherence reporting in specialty pharmacy. J Manag Care Spec Pharm. 2019;25(10):1073-77. doi:10.18553/ jmcp.2019.25.10.1073

7. Centers for Medicare \& Medicaid Services. 2021 Medicare Part C \& D star ratings technical notes. October 1, 2020. Accessed August 12, 2021. https://www.cms.gov/files/ document/2021technotes20201001.pdf-0

8. Utilization Review Accreditation Commission. 2021 drug therapy management measures at a glance. Updated December 15, 2020.

Accessed August 12, 2021. https:// www.urac.org/accreditation-cert/ drug-therapy-management-accreditation/

9. McCabe CC, Barbee MS, Watson ML, et al. Comparison of rates of adherence to oral chemotherapy medications filled through an internal health-system specialty pharmacy vs external specialty pharmacies. Am J Health Syst Pharm. 2020;77(14):1118-27. doi:10.1093/ajhp/ zxaa135

10. Schwartz RN, Eng KJ, Frieze DA, et al. NCCN task force report: specialty pharmacy. J Natl Compr Canc Netw. 2010;8(Suppl 4):S1-S12. doi:10.6004/ jnccn.2010.0127

11. Farano JL, Kandah H-M. Targeting financial toxicity in oncology specialty pharmacy at a large tertiary academic medical center. J Manag Care Spec Pharm. 2019;25(7):765-69. doi:10.18553/ jmcp.2019.25.7.765

12. Choudhry NK, Shrank WH, Levin RL, et al. Measuring concurrent adherence to multiple related medications. Am J Manag Care. 2009;15(7):457-64.
13. Steiber D. Community specialty pharmacy: the time is now! Specialty Pharmacy Times. July/August 2014. Volume 5, Issue 4. Accessed December 25, 2020. https://www.pharmacytimes.com/ view/community-specialty-pharmacythe-time-is-now

14. Hagerman J, Freed S, Rice G. Specialty pharmacy: a unique and growing industry. Pharmacy Today. 2013;19(7):P31. Accessed August 12, 2021. https://www.pharmacytoday.org/article/S1042-0991(15)31266-4/ fulltext\#relatedArticles

15. Adelson KB, Stutsky M, Fradkin M, et al. Abstract 108: Should cancer centers start their own specialty pharmacy? Quality and economic data from the oral chemotherapy program at Smilow Cancer Hospital and Yale New Haven Health System. J Clin Oncol. 2017;35(8 Suppl):108. doi: 10.1200/JCO.2017.35.8_suppl.108

16. National Cancer Institute, Surveillance, Epidemiology, and End Results Program. Cancer stat facts: colorectal cancer. Accessed May 17, 2021. https://seer.cancer.gov/statfacts/html/colorect.html

17. National Cancer Institute, Surveillance, Epidemiology, and End Results Program. Cancer stat facts: ovarian cancer. Accessed May 17, 2021. https://seer.cancer.gov/statfacts/html/ovary.html

18. Mislang AR, Wildes TM, Kanesvaran R, et al. Adherence to oral cancer therapy in older adults: the International Society of Geriatric Oncology (SIOG) task force recommendations. Cancer Treat Rev. 2017;57:58-66. doi:10.1016/j. ctrv.2017.05.002

19. Gandhi S, Day L, Paramsothy T, et al. Oral anticancer medication adherence, toxicity reporting, and counseling: a study comparing health care providers and patients. J Oncol Pract. 2015;11(6):498-504. doi:10.1200/jop.2015.004572 